\title{
Coalition Network Elements for Base Station Cooperation
}

\author{
Jie Zhang ${ }^{\dagger *}$, Rong Zhang*, Guangjun Li ${ }^{\dagger}$ and Lajos Hanzo* \\ $\dagger$ School of Comm. Info. and Engineering, UESTC, China. $*$ School of ECS., Univ. of Southampton, UK. \\ Email: jz4,rz,lh@ecs.soton.ac.uk, http://www-mobile.ecs.soton.ac.uk
}

\begin{abstract}
Coalition Network Elements (CNE) are proposed for Base Stations (BS) cooperation, where the CNEs carry traffic for the BS in support of its cell-edge MSs by exploiting the unused frequency bands of the BS network, while considering a range of practical impairments. We derive the coalition probability by taking into account both system loads of the primary network as well as the CNE's greediness. Our simulation results demonstrate that the proposed solution is capable of substantially increasing the attainable SINR in a wide range of scenarios and it is also robust to diverse practical imperfections.
\end{abstract}

\section{INTRODUCTION}

In the family of Co-Channel Interference (CCI) mitigation techniques [1], the Multiple-Input Multiple-Output (MIMO) based BS-cooperation constitutes a promising enabler in future wireless access networks, hence it has been investigated in both academia and industry [2]-[4]. However, the original Downlink (DL) BS cooperation requires full Channel State Information (CSI) of all links between all BSs and MSs at the transmitter side for approaching the theoretical upper-bound performance. Naturally, the presence of imperfect and outdated CSI at the cooperative BS transmitters will erode the efficiency of this CCI mitigation technique.

A classic solution conceived for mitigating the hostile channel-effects is to employ Automatic Repeat reQuest (ARQ) type retransmissions from all of the cooperative BSs. However, full ARQ-aided BS-Cooperation may impose a high overhead, yet the overall gain may remain limited. Furthermore, the inherent delay of the ARQ-aided BS-Cooperation potentially limits their employment to delay-intolerant scenarios. As a different design alternative, Relay Stations (RS) may be installed in the cell-edge area for the sake of providing another dedicated source of diversity for a specific BS to MS link. In contrast to ARQ-aided BS-Cooperation, which relies on the same DL BS transmitters, RS-aided BS-Cooperation relies on two different DL transmitters. The RS is effective in covering potential blind spots, but it may aggregate the CCI in the celledge area of the adjacent cells. Furthermore, most of the RS research considered altruistic relaying, where the RS provides services for free as an integrated part of the network.

In contrast to the above two solutions, our novel contribution is that we further expand the concept of coordinated multipoint transmissions, and propose remote Coalition Network Elements (CNE) for the high-integrity coverage of the celledge area, where the BSs cooperatively transmit to the celledge MSs during the first hop, while the CNE is responsible for the complementary second-hop transmission. The following aspects highlight the novel properties of the proposed CNE:

1) The features of CNEs: In contrast to the conventional altruistic RSs, which convey information for the source for free, the CNEs 'borrowed' from different networks will selfishly tap into some of the already allocated

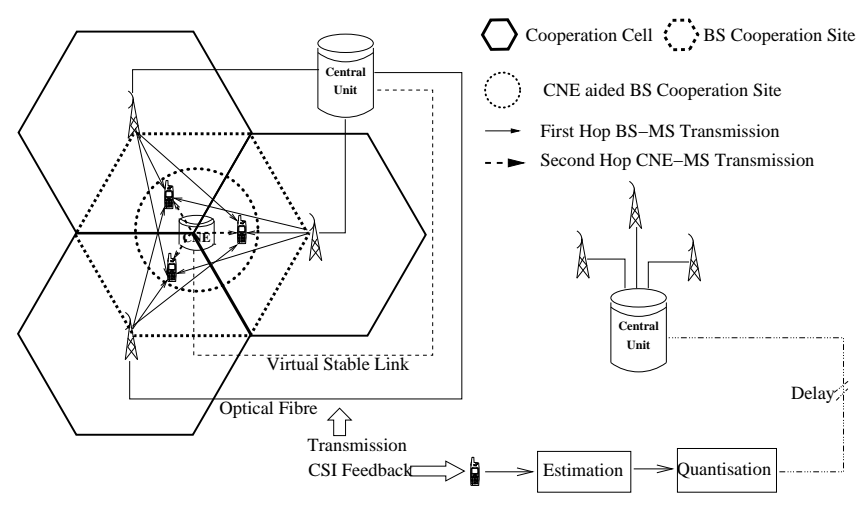

Fig. 1. The cellular topology considered and CSI feedback process

resources for the sake of conveying their own traffic, which we may refer to as a form of coalition.

2) The operation of CNEs: The CNE carries traffic for the BSs in the unutilised frequency bands of the BS network, where the availability of these idle channels is explicitly signalled by the cooperative BSs, rather than being cognitively sensed. As a result, the CNE may act as a fall-back solution in support of BS-cooperation, when the BSs suffers from grave impairments.

We organise our paper as follows. In Section II, our system model and assumptions are provided. Then, the noncooperation, conventional BS-cooperation, ARQ-aided BScooperation and CNE-aided BS-cooperation are discussed in Section III. The system performance is investigated in Section IV. Finally, we conclude in Section V. ${ }^{1}$

\section{SYSTEM DESCRIPTION}

\section{A. Cellular Topology}

Let us first introduce the cellular topology of Fig. 1, where the hexagonal cellular model associated with Unity Frequency Reuse (UFR) is employed. As shown in Fig.1, three BSs form a joint cooperative transmission site, where three MSs supported by each of the anchor BSs are involved in the joint transmission. Hence the cell-edge of the conventional cells effectively becomes the cell-centre of the newly formed cooperative site, as indicated by the circle in Fig. 1. In the centre of the cooperative site, a remote $\mathrm{CNE}$ is introduced working in coalition, which may belong to the same operator but may be assigned to a different service mission or to other network operators.

Consider a general CNE-aided BS-Cooperation scenario constituted by $N_{b}$ BSs and one CNE, where each BS is equipped with $N_{t}$ transmit antennas. Let us assume that a total of $N_{u}$ MSs - each equipped with $N_{r}$ receive antennas - are

\footnotetext{
${ }^{1}$ Notation: Throughout the paper, the superscript $(\cdot)^{T}, \mathbf{E}(\cdot)$ and trace $(\cdot)$ denote the transpose, the expectation and the trace operation, respectively.
} 
involved in the cooperative transmission, where each of the $N_{u}$ MSs roams within the coverage area of its anchor BS, as portrayed in Fig. 1. We let $N_{t}^{c}$ denote the number of transmit antennas at the CNE. Furthermore, we let $N_{T}=\left(N_{b} \times N_{t}\right)$ and $N_{R}=\left(N_{u} \times N_{r}\right)$ denote the total number of transmit and receive antennas in the $\mathrm{BS}$ cooperation phase, respectively.

\section{B. Configurations and Assumptions}

Within the two-hop transmission scenario considered, the assumption of near-perfect reception of the BSs' data at the CNE becomes realistic, when an optical fibre link or a microwave backhaul link benefiting from directional antennas and strong Line-of-Sight (LoS) propagation is used for connecting the cooperative BSs and the CNE. Since the novelty of this paper is the introduction of the CNEs, we consider the following configurations for ease of exposition. We assume that the number of receive antennas at each MS is $N_{r}=1$. This allows us to avoid the complications of joint transmitter and receiver design, which is set aside for our future work. We also assume that the number of transmit antennas at the CNE satisfies $N_{t}^{c}=1$, which avoids advanced space-time processing at the CNE's side.

In order to compare the proposed CNE-aided BSCooperation to both conventional BS-Cooperation and to ARQ-aided BS-Cooperation, the total power consumption $P_{t}$ is assumed to be the same for the sake of a fair comparison. More explicitly, we let $P_{\mathbb{B}_{c}}=P_{t}$ in the conventional BSCooperation, while we set $P_{\mathbb{B}_{c}}^{(1)}+P_{\mathbb{B}_{c}}^{(2)}=P_{t}$ in the ARQaided BS-Cooperation and $P_{\mathbb{B}_{c}}+P_{C}=P_{t}$ in the proposed CNE-aided BS-Cooperation, where $P_{\mathbb{B}_{c}}$ and $P_{C}$ denote the transmission power of all cooperative BSs and that of the CNE, respectively. Furthermore, $P_{\mathbb{B}_{c}}^{(1)}$ and $P_{\mathbb{B}_{c}}^{(2)}$ denote the transmission power of the first- and second-attempt in ARQ-aided BSCooperation. In this study, we dispense with optimising the power allocation and rely on the equal-power assumption for the three cooperative BSs and the CNE.

\section{CNE-AIDED BS COOPERATIVE DL TRANSMISSION}

\section{A. Benchmarker Scenario}

1) Non-Cooperative Transmission: In this scenario, each MS is only served by its anchor BS, while the remaining active transmissions are considered as CCI. The discrete-time model of the signal received by MS $j$ may be written as:

$$
y_{j}=\mathbf{h}_{j, j} \mathbf{t}_{j, j} x_{j}+\sum_{i \in \mathbb{B}_{c,-j}} \mathbf{h}_{i, j} \mathbf{t}_{i, i} x_{i}+n_{j},
$$

where the variable $n_{j}$ denotes the Gaussian noise having a covariance of $N_{0}$. The vector $\mathbf{h}_{i, j} \in \mathbb{C}^{1 \times N_{t}}$ describes the DL Multiple-Input Single-Output (MISO) channel between the $i$ th BS and the $j$ th MS, while $\mathbf{t}_{j, j}=\sqrt{P_{B} / N_{t}}[1,1, \cdots, 1]^{T} \in$ $\mathbb{C}^{N_{t} \times 1}$ denotes the preprocessing employed at the $j$ th BS for transmitting the $j$ th MS's signal. Furthermore, $x_{j}$ denotes the transmitted signal intended for MS $j$, which is independent of both the noise and of the channel, obeying $\mathbf{E}\left(x_{j} x_{j}^{*}\right)=1$. Thus, the Signal-to-Interference-plus-Noise-Ratio (SINR) of MS $j$ may be expressed as:

$$
\gamma_{j}^{s c p} \geq \frac{P_{B} \mathbf{E}\left(\left|\mathbf{h}_{j, j}\right|^{2}\right) / N_{t}}{N_{0}+P_{B} \sum_{i \in \mathbb{B}_{c,-j}} \mathbf{E}\left(\left|\mathbf{h}_{i, j}\right|^{2}\right) / N_{t}},
$$

where the inequality is derived from Jensen's Inequality.
2) BS Cooperation: In a BS-cooperation scenario, each MS is jointly served by all BSs of the cooperative site. Hence, the discrete-time model of MS $j$ may be written as:

$$
y_{j}=\mathbf{h}_{j} \mathbf{t}_{j} x_{j}+\sum_{i \in \mathbb{B}_{u,-j}} \mathbf{h}_{j} \mathbf{t}_{i} x_{i}+n_{j},
$$

where the first two terms represent the desired signal and the DL Multi-User Interference (MUI) that is imposed by the simultaneous transmissions to other MSs in the cooperative site. The difference in comparison to the system model of the noncooperative transmission is that $\mathbf{h}_{j}=\left[\mathbf{h}_{1, j}, \mathbf{h}_{2, j}, \ldots, \mathbf{h}_{N_{b}, j}\right]$ denotes the joint channel between all the $N_{b}$ cooperative BSs and the $j$ th $\mathrm{MS}$, with $\mathbf{H}=\left[\mathbf{h}_{1}^{T}, \mathbf{h}_{2}^{T}, \ldots, \mathbf{h}_{N_{u}}^{T}\right]^{T}$ denoting the channel matrix. Here, $\mathbf{t}_{j} \in \mathbb{C}^{N_{T} \times 1}$ denotes the joint precoding vector of the cooperative site intended for MS $j$ and $\mathbf{T}=\left[\mathbf{t}_{1}, \mathbf{t}_{2}, \ldots, \mathbf{t}_{N_{u}}\right] \in \mathbb{C}^{N_{T} \times N_{R}}$ acts as the precoding matrix of the cooperative site.

Let us now introduce a simple equal-powerallocation scheme associated with diagonal matrix $\mathbf{G}=\operatorname{diag}\left(\sqrt{P_{\mathbb{B}_{c}} / N_{u}}, \cdots, \sqrt{P_{\mathbb{B}_{c}} / N_{u}}\right) \in \mathbb{R}^{N_{u} \cdot N_{s} \times N_{u} \cdot N_{s}}$ employed in our DL BS cooperation scenario, obeying the total BS power constraint of $\sum_{j=1}^{N_{u}}$ trace $\left(\mathbf{t}_{j} \mathbf{t}_{j}^{H}\right)=P_{\mathbb{B}_{c}}$. In this paper, we employ the classic Zero-Forcing (ZF) DL linear precoding technique, which is capable of completely eliminating the MUI, provided that perfect CSI is available at the transmitters. The precoding matrix $\mathbf{T}$ may be written as $\mathbf{T}=\mathbf{W G}$, where the matrix $\mathbf{W}$ has normalised column vectors which is designed by $\mathbf{H}^{H}\left(\mathbf{H H}^{H}\right)^{-1}$ [3]. Hence, the SINR of the $j$ th MS may be expressed as:

$$
\gamma_{j}^{m c p} \geq \frac{P_{\mathbb{B}_{c}} \mathbf{E}\left(\left|\mathbf{h}_{j} \mathbf{w}_{j}\right|^{2}\right) / N_{u}}{N_{0}+P_{\mathbb{B}_{c}} \sum_{i \in \mathbb{B}_{u,-j}} \mathbf{E}\left(\left|\mathbf{h}_{j} \mathbf{w}_{i}\right|^{2}\right) / N_{u}},
$$

where the above expectations $\mathbf{E}\left(\left|\mathbf{h}_{j} \mathbf{w}_{j}\right|^{2}\right)$ and $\mathbf{E}\left(\left|\mathbf{h}_{j} \mathbf{w}_{i}\right|^{2}\right)$ are provided by the following Lemma 3.1.

Lemma 3.1: With considering practical CSI impairments of Appendix A, $\mathbf{E}\left(\left|\mathbf{h}_{j} \mathbf{w}_{j}\right|^{2}\right)$ and $\mathbf{E}\left(\left|\mathbf{h}_{j} \mathbf{w}_{i}\right|^{2}\right)$ is given by

$$
\begin{aligned}
& \mathbf{E}\left(\left|\mathbf{h}_{j} \mathbf{w}_{j}\right|^{2}\right)= \rho^{2} \delta\left[(1-\varphi)\left(N_{T}-N_{u}+1\right) / N_{T}+\varphi / N_{T}\right] \\
&+\rho^{2} \sigma_{e}^{2}+1-\rho^{2}, \\
& \mathbf{E}\left(\left|\mathbf{h}_{j} \mathbf{w}_{i}\right|^{2}\right)= \rho^{2} \delta \varphi /\left(N_{T}-1\right)+\rho^{2} \sigma_{e}^{2}+1-\rho^{2} . \\
& \quad \text { Proof: See Appendix B. }
\end{aligned}
$$

3) ARQ-Aided BS-Cooperation: If the BS-Cooperation fails to meet the target integrity at the MSs, the MSs in the celledge area may request ARQ-aided retransmission, which may be encountered owning to a range of practical impairments. Hence, in this straightforward setting, the discrete-time model of the DL received signal at MS $j$ in each of the two attempts may be written as Eq. (2), while obeying the total power constraint of $P_{\mathbb{B}_{c}}^{(1)}=P_{\mathbb{B}_{c}}^{(2)}=P_{t} / 2$. Likewise, the average SINR of MS $j$ using the Maximal Ratio Combining (MRC) criterion may be formulated as:

$$
\gamma_{j}^{a} \geq \frac{0.5 P_{t} / N_{u}\left[2 \mathbf{E}\left(\left|\mathbf{h}_{j} \mathbf{w}_{j}\right|^{2}\right)\right]^{2}}{2 N_{0} \mathbf{E}\left(\left|\mathbf{h}_{j} \mathbf{w}_{j}\right|^{2}\right)+P_{t} / N_{u} \sum_{i \neq j} \mathbf{\Xi}_{j, i}},
$$

where $\boldsymbol{\Xi}_{j, i}=\mathbf{E}\left(\left|\mathbf{h}_{j} \mathbf{w}_{j}\right|^{2}\right) \mathbf{E}\left(\left|\mathbf{h}_{j} \mathbf{w}_{i}\right|^{2}\right)$, while $\mathbf{E}\left(\left|\mathbf{h}_{j} \mathbf{w}_{j}\right|^{2}\right)$ and $\mathbf{E}\left(\left|\mathbf{h}_{j} \mathbf{w}_{i}\right|^{2}\right)$ are provided by Lemma 3.1.

\section{B. CNE-Aided BS-Cooperative DL Transmission}

In contrast to the above-mentioned closed-loop ARQ-aided BS-Cooperation, the CNE proposed may be operated in an 
open-loop manner for the second-hop transmission. The transmissions from the CNE to the MSs take place in unutilised frequency bands of the primary BS network, which are allocated by the cooperative BSs and are then conveyed to the $\mathrm{CNE}$ in an active notification manner.

1) SINR of CNE-Aided BS-Cooperation: In our proposed scenario, the CNE will serve the MSs in a round-robin fashion, when there are insufficient frequencies to be assigned to the CNE for serving all cell-edge MSs. The received signal of the first hop is given by Eq. (2) and the signal received from the second hop is $y_{j, k}^{c}=\sqrt{P_{C} / k} h_{j}^{c} x_{j}+n_{j}^{c}$, where $k$ denotes the number of idle channels available for the secondhop transmission. Hence, the corresponding SINR of MS $j$ benefitting from a second-hop transmission is given by

$$
\gamma_{j, k}^{c} \geq \frac{\left|\sqrt{P_{\mathbb{B}_{c}} / N_{u}} \mathbf{E}\left(\left|\mathbf{h}_{j} \mathbf{w}_{j}\right|^{2}\right)+\sqrt{P_{C} / k} \mathbf{E}\left(\left|h_{j}^{c}\right|^{2}\right)\right|^{2}}{\mathbf{E}\left(\left|\mathbf{h}_{j} \mathbf{w}_{j}\right|^{2}\right) N_{0}+\mathbf{E}\left(\left|h_{j}^{c}\right|^{2}\right) N_{0}+P_{\mathbb{B}_{c}} / N_{u} \sum_{i \neq j} \mathbf{\Xi}_{j, i}} .
$$

Let us now derive the probability of having $k$ unutilised channels available for the CNE. We let $N_{c}$ and $N_{c}^{b}$ denote the number of total channels and the number of busy channels in each of the cooperating cells, respectively. We define the socalled system load as $\beta=N_{c}^{b} / N_{c}, \beta \in[0,1]$ and introduce the factor of greediness for the CNE as $\eta \in[0,1]$, which implies that the CNE would reserve a fraction $\eta$ of the available channels for its own transmissions. The probability of having $k$ idle channels unoccupied by any coooperative BS for the CNE's promised second-hop transmission may be written as

$$
p(k)=\left(\begin{array}{c}
N_{c} \\
\left\lceil\frac{k}{1-\eta}\right\rceil
\end{array}\right)\left[(1-\beta)^{N_{b}}\right]^{\left\lceil\frac{k}{1-\eta}\right\rceil}\left[1-(1-\beta)^{N_{b}}\right]^{N_{c}-\left\lceil\frac{k}{1-\eta}\right\rceil},
$$

with $[\cdot]$ denoting the ceiling operator. Thus, the average SINR of the CNE-aided BS-cooperation may be formulated as

$$
\gamma^{c}=p(0) \gamma_{j}^{m c p}+\sum_{k=1,2, \geq 3} p(k) \gamma_{j, k}^{c} \min \left(k / N_{u}, 1\right)
$$

2) Feasibility of CNE-Aided BS-Cooperation: Let us firstly introduce some further notations required for our discussions. We let $\alpha=1-e^{-a \lambda}$ [5] denote the probability of the realisation of a particular pricing $p$, where $\lambda=\ln (\phi)-p \phi$ represents the end-user utility score. Additionally, we let $\phi=$ $\ln (\gamma)$ denote the bandwidth efficiency per channel provided by a particular technology, while $N_{c}^{o}, N_{c}^{o 1}$ and $N_{c}^{o 2}$ denote the number of unutilised channels in the cooperative site, the number of channels used by the CNE for carrying the main BSs' traffic and the number of channels reserved for the CNE's own usage, respectively. Furthermore, the subscripts $(\cdot)_{0},(\cdot)_{1}$, $(\cdot)_{2}$ represent the original stand-alone BS-Cooperation, the BS network supported by CNE and the CNE network itself. Finally, $p_{L}$ denotes the leasing price of the frequency bands.

The profit of the original stand-alone BS-Cooperation arrangement supporting a full system load is given by:

$$
\mathcal{Q}_{0}=\alpha\left(\phi_{0}, p_{0}\right) \phi_{0} N_{c} p_{0} .
$$

The profit of the BS network supported by CNE is given by

$$
\mathcal{Q}_{1}=\alpha\left(\phi_{1}, p_{1}\right) \phi_{1} N_{c}^{o 1} p_{1}+\alpha\left(\phi_{0}, p_{1}\right) \phi_{0} N_{c}^{b} p_{1}+N_{c}^{o} p_{L},
$$

where the first term represents the profit made as a result of coalition, the second term is the profit of using the original technology and the third term denotes the leasing income. The profit of the CNE network as a result of this coalition is

$$
\mathcal{Q}_{2}=\alpha\left(\phi_{2}, p_{2}\right) \phi_{2} N_{c}^{o 2} p_{2}-N_{c}^{o} p_{L},
$$

where the first term is a result of utilising the reserved channels and the second term is the loss due to leasing. The objective of establishing the pricing model is to find the optimum values of $p_{0}^{o}, p_{1}^{o}, p_{2}^{o}$ such that both the BSs and the CNE agree to a certain leasing price $p_{L}$ and the sum profit of $\mathcal{Q}=\mathcal{Q}_{1}+\mathcal{Q}_{2}-$ $\mathcal{Q}_{0}$ is positive. In order to arrive at this, we use an iterative procedure for exchanging information between the following profit maximisation step and the price negotiation step.

a) Profit Maximisation: This step is executed by assuming that the leasing price $p_{L}$ has already been agreed. In order to solve the profit maximisation problem, we take first derivative of the cooresponding profit of Eq. (3), Eq. (4), Eq. (5) with respect to $p_{0}, p_{1}, p_{2}$ and solve the resultant equations by using a standard numerical equation solver.

b) Price Negotiation: Note that the condition for a CNE to participate in the coalition is that its profit obeys $\mathcal{Q}_{2}>0$ and the condition for the main BS network to invite a coalition partner is that of $\mathcal{Q}_{1}>\mathcal{Q}_{0}$. For the former condition, we can find an upper bound $p_{L}^{u . b}$ for the leasing price, while for the latter condition, we can find a lower bound $p_{L}^{l . b}$ for the leasing price. As a result, we may conclude that a system equilibrium was found when $p_{L}^{l . b}<p_{L}<p_{L}^{u . b}$.

\section{Performance Evaluation}

In this section, we consider a practical CNE-aided BScooperation system, consisting of three cooperative BSs, three MSs and one CNE. Here, we considered the Urban Micro setup [6], where the BS-to-BS distance and the BS radius were defined as $D=1000 \mathrm{~m}$ and $R=D / \sqrt{3}$, respectively. The channel of each BS-MS pair and of each CNE-MS pair are constituted by three components, i.e. by $\mathbf{h}_{i, j}=$ $\left(A_{i, j}^{p l} A_{i, j}^{s}\right)^{1 / 2} \mathbf{h}_{i, j}^{f}$, where $\mathbf{h}_{i, j}^{f} \in \mathbb{C}^{N_{r} \times N_{t}}$ represents the fast fading component, which is assumed to be frequency-flat with zero-mean and unity-variance complex Gaussian entries, while $A_{i, j}^{s}=10^{\xi / 10}$ is the lognormal shadow fading component, where $\xi$ is generated by a zero-mean real-valued Gaussian random variable having a standard derivation of $\sigma_{s}=8 \mathrm{~dB}$. The pathloss model is given by $A_{i, j}^{p l}=\beta^{p l} d_{i, j}^{\alpha^{p l}}$, where $d_{i, j}$ denotes the distance in meter between the $i$ th BS and $j$ th MS, while we have $\left[\alpha^{p l} ; \beta^{p l}\right]=\left[-3 ; 1.35 \times 10^{7}\right]$ [7]. We investigate the SINR of the MSs that are located on a circle with radii of $r=\{0.1 R, 0.2 R\}$ centred at the CNE.

\section{A. Investigations of SINR}

Fig. 2(a) illustrates the achievable SINR per user at radii $\{0.1 R, 0.2 R\}$ of different BS cooperation arrangements corresponding to a greedy factor of $\eta=0.4$ and to a system load of $\beta=\{0.6,0.8\}$. From the figure, we can observe that the SINR of our proposed solution is superior in comparison to both the ARQ-aided BS-cooperation, to the conventional BScooperation as well as to the non-cooperative benchmarker right across the entire SNR range for both a high system load of $\beta=0.8$ as well as for a moderate system load of $\beta=0.6$. This is achieved by exploiting the idle channels of the primary BS network and the shorter, better-quality links between the $\mathrm{CNE}$ and the users during the second-hop transmission. 


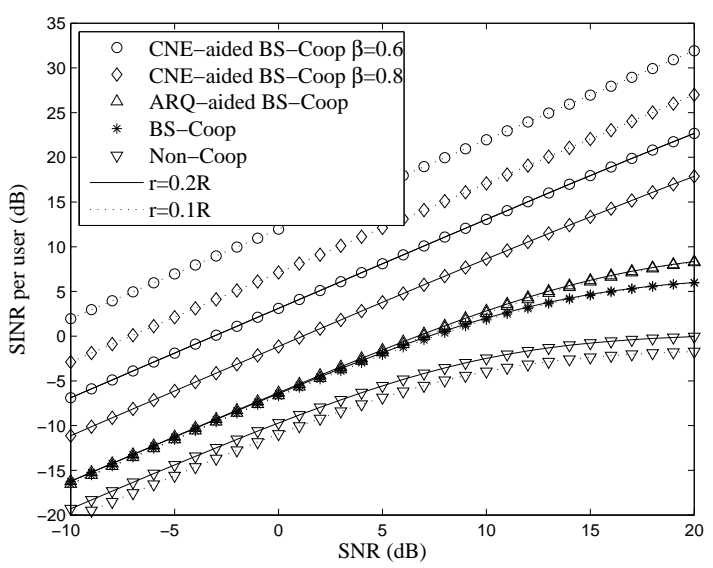

(a) SINR versus SNR
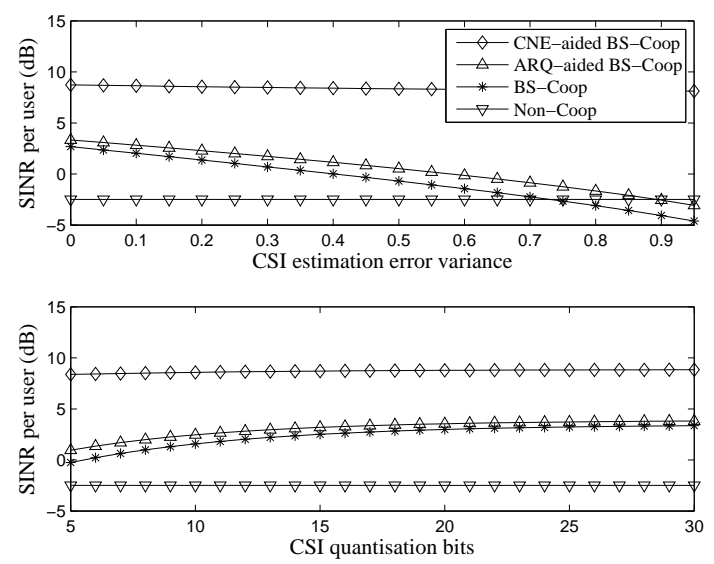

(c) SINR with different CSI errors
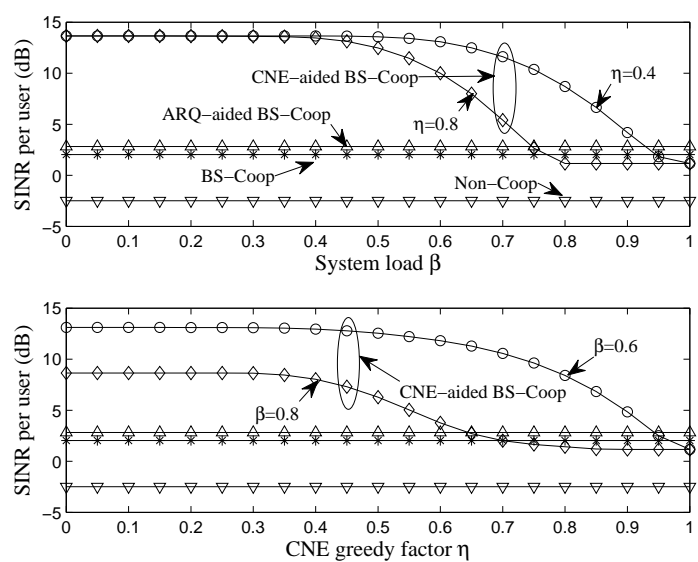

(b) SINR vs. system loads and greedy factors

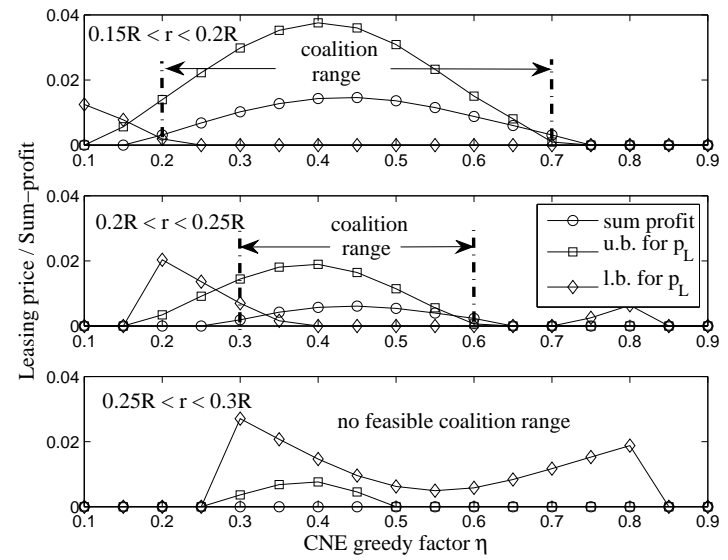

(d) Feasibility of coalition

Fig. 2. Fig. 2(a): SINR per user versus SNR at a greedy factor of 0.4 , when the radii are $r=\{0.1 R, 0.2 R\}$. Fig. 2 (b): SINR for different system loads and greedy factors, when the radius is $r=0.2 R$ and $N_{c}=32$. Fig. 2(c): SINR vs. CSI estimation error variance and vs. CSI quantisation bits, with $r=0.2 R$, $\beta=0.8, \eta=0.4$ and $N_{c}=32$. Fig. 2(d): Feasible coalition range of the greedy factors for system load $\beta=0.8$, available channels $N_{c}=32$ and acceptance ratio $a=0.5$.

Furthermore, Fig. 2(a) shows that the SINR of our proposed solution has an inversely proportional relation to the radius $r$, namely the lower $r$, the higher the achievable SINR. Additionally, we may also observe the plausible fact that the lower the system load $\beta$, the better the SINR becomes, owing to the fact that more idle channels are available for the secondhop transmissions within a low-load system.

\section{B. Effects of System Load and Greedy Factor}

With considering the effects of the system load and greedy factor, both the non-cooperative transmission, as well as the BS-Cooperation and the ARQ-aided BS-Cooperation remain unaffected, because there are always sufficient channels for supporting the offered traffic. However, the performance of the proposed scenario is sensitive to both the system load and to the greedy factor, as shown in Fig. 2(b) at a SNR of 10dB.

As shown in the upper figure of Fig. 2(b), the SINR of the proposed scenario is higher than that of all the other three transmission scenarios, with the exception of high system load scenarios. Specifically, when the system load is in the range of $\beta \in[0,0.4]$, there is no obvious erosion of the achievable SINR, since sufficient idle channels are available in the primary BS network for supporting the second-hop transmissions. However, when $\beta>0.4$ is considered, the availability of idle channels becomes less likely, hence the achievable SINR of the proposed solution decays rapidly. Finally, the SINR of the proposed solution becomes worse than that of the conventional BS-Cooperation ${ }^{2}$. As for the effects of the greedy factor, similarly, there is no significant SINR degradation, when the greedy factor increases from $\eta=0$ to $\eta=0.4$. Beyond this threshold, the SINR begins to decay and ultimately becomes lower than that of the ARQ-aided scheme and that of the conventional BS-Cooperation regime, as $\eta$ approaches one, in which case the CNE reserves all assigned channels for the sake of conveying its own traffic, i.e. without transmitting any of the source's traffic to the cell-edge users.

\section{Effects of Practical Impairments}

Fig. 2(c) quantifies the achievable SINR as a function of the CSI estimation error's variance (upper subfigure) and of the CSI quantisation error (lower subfigure) at an SNR of $10 \mathrm{~dB}$, respectively. We initially assume having no impairments in the

${ }^{2}$ In fact, the worst SINR that can be achieved by our CNE-aided BSCooperation should be the same as that of the conventional BS-Cooperation since the CNE-aided BS-Cooperation degenerates into conventional BSCooperation. In Fig. 2(b), the degraded SINR is however achievable at $75 \%$ of the transmit power assigned to the first BS to MS transmission in comparison to the conventional BS-Cooperation. 
non-cooperative DL transmission scenario, which is then used as the benchmarker. The interference mitigation capability of the BS cooperation technique is heavily dependent on the accuracy of the precoding matrix, which is the function of the instantaneous CSI accuracy. Hence as expected, the SINR curves decay, when the variance $\sigma_{e}^{2}$ of the CSI estimation error increases. This phenomenon is observed for the conventional BS-Cooperation, for the ARQ-aided BS-Cooperation and for the proposed solution. Furthermore, the SINR of the proposed scenario relies both on the first-hop and on the second-hop open-loop transmission, where the latter is unaware of the CSI errors, hence the SINR of the proposed scenario suffers from a reduced degradation. As a result, the proposed scenario maintains a consistently higher SINR than that of the ARQaided and that of the conventional BS-Cooperation.

\section{Feasibility Study}

Fig. 2(d) investigates the effects of the greedy factor $\eta$ on the proposed scenario for different coverage ranges. Note that the feasibility range is deemed to be where the sum-profit is positive, which happens only when $p_{L}^{l . b}<p_{L}<p_{L}^{u . b}$ holds. Observe in Fig. 2(d) that the coalition range is shrunk, when the MSs are located further away from the CNE and the feasibility of this coalition is diminishing for $0.25 R<$ $r<0.3 R$. Furthermore, the sum-profit of BSs and the CNE reached its peak as the greedy factor is at about 0.45 , as shown in the top and middle subplot of Fig. 2(d).

\section{CONCLUSIONS}

We proposed a CNE-aided BS-cooperation transmission scenario employing the $\mathrm{ZF}$ precoding technique, where the greedy CNE cooperates with the primary BSs. The sensitivity of the system performance was characterised as a function of both the system load and of the greedy factor. Our numerical results demonstrated that the proposed scenario achieves a significant SINR improvement across a large fraction of the coverage area in the presence of practical CSI impairments.

\section{APPENDIX}

\section{A. Practical Impairments}

1) CSI Estimation Errors: We assume that MS $j$ is capable of estimating the joint CSI vector $\mathbf{h}_{j}$, subject to the assumption of a Gaussian CSI estimation error having a variance of $\sigma_{e}^{2}$. Then the channel vector of MS $j$ at the time of estimation may be expressed as $\mathbf{h}_{j}=\hat{\mathbf{h}}_{j}+\sigma_{e} \mathbf{e}_{j}$, where $\mathbf{e}_{j}$ denotes a zero-mean, unit-variance complex Gaussian vector.

2) CSI Quantisation Errors: Have obtained the estimated CSI, the Random Vector Quantisation (RVQ) scheme [8] is employed for quantising the Channel Direction Information (CDI) $\tilde{\mathbf{h}}_{j}=\hat{\mathbf{h}}_{j} /\left|\hat{\mathbf{h}}_{j}\right|$, where $\left|\hat{\mathbf{h}}_{j}\right|$ represents the Channel Quality Information (CQI). This implies that a CDI quantisation codebook $\mathbf{C}=\left\{\mathbf{c}_{1}, \mathbf{c}_{2}, \ldots, \mathbf{c}_{N_{q}}\right\}$ consisting of $N_{q}=2^{b}$ vectors is constructed and made available to both the MS and to the cooperative BSs. In the quantised feedback regime, the particular codebook index of $\kappa_{j}=\max _{q} \cos \theta, \theta=\angle\left(\tilde{\mathbf{h}}_{j}, \mathbf{c}_{q}\right)$ are transmitted. Then, we have $\tilde{\mathbf{h}}_{j}=\mathbf{c}_{\kappa_{j}} \cos \theta+\mathbf{g}_{\kappa_{j}} \sin \theta$ and $\varphi=\mathbf{E}\left(\sin ^{2} \theta\right)=$ $2^{b} \Gamma\left(2^{b}\right) \Gamma\left[N_{T} /\left(N_{T}-1\right)\right] / \Gamma\left[2^{b}+N_{T} /\left(N_{T}-1\right)\right]$, where $\mathbf{g}_{\kappa_{j}}$ and $\Gamma(x)$ denote a unit vector that lies in the null-space of $\mathbf{c}_{\kappa_{j}}$ and the Gamma function, respectively.
3) CSI Feedback Delays: When using Jake's model, the channel vector $\mathbf{h}_{j}$ of the $j$ th MS can be written in the presence of all three practical CSI impairments as

$$
\mathbf{h}_{j}=\rho\left[\left|\hat{\mathbf{h}}_{j}\right|^{2}\left(\mathbf{c}_{\kappa_{j}} \cos \theta+\mathbf{g}_{\kappa_{j}} \sin \theta\right)+\sigma_{e} \mathbf{e}_{j}\right]+\sqrt{1-\rho^{2}} \mathbf{z}_{j},
$$

where $\rho=J_{0}\left(2 \pi f_{d} \tau\right)$ and $J_{0}(\cdot)$ denotes the zero-order Bessel function of the first kind, while $f_{d}$ and $\tau$ denote the maximum Doppler frequency shift and feedback delay, respectively. Finally, $\mathbf{z}_{j}$ denotes a zero-mean, unit-variance complex Gaussian vector.

\section{B. Proof of Lemma 3.1}

The channel vector $\mathbf{h}_{j}$ is given by Eq. (6), thus the quantity $\left|\mathbf{h}_{j} \mathbf{w}_{j}\right|^{2}$ can be expanded as:

$$
\begin{aligned}
\left|\mathbf{h}_{j} \mathbf{w}_{j}\right|^{2} & =\rho^{2}\left|\hat{\mathbf{h}}_{j}\right|^{2} \cos ^{2} \theta\left|\mathbf{c}_{\kappa_{j}} \mathbf{w}_{j}\right|^{2}+\rho^{2}\left|\hat{\mathbf{h}}_{j}\right|^{2} \sin ^{2} \theta\left|\mathbf{g}_{\kappa_{j}} \mathbf{w}_{j}\right|^{2} \\
& +\rho^{2} \sigma_{e}^{2}\left|\mathbf{e}_{j} \mathbf{w}_{j}\right|^{2}+\left(1-\rho^{2}\right)\left|\mathbf{z}_{j} \mathbf{w}_{j}\right|^{2}
\end{aligned}
$$

When using the $\mathrm{ZF}$ precoding criterion, $\mathbf{w}_{j}$ lies in the nullspace of $\mathbf{c}_{\kappa_{j}}^{\perp}=\left\{\mathbf{c}_{1}, \mathbf{c}_{2}, \ldots, \mathbf{c}_{\kappa_{j}-1}, \mathbf{c}_{\kappa_{j}+1}, \ldots, \mathbf{c}_{N_{u}}\right\}$. The quantity $\left|\mathbf{c}_{\kappa_{j}} \mathbf{w}_{j}\right|^{2}$ is the square norm of the projection of $\mathbf{c}_{\kappa_{j}}$ on $\mathbf{w}_{j}$ in the $\left(N_{T}-N_{u}+1\right)$ dimensional space. From [9], we have $\left|\mathbf{c}_{\kappa_{j}} \mathbf{w}_{j}\right|^{2} \sim \chi_{2\left(N_{T}-N_{u}+1\right)}^{2},\left|\mathbf{e}_{j} \mathbf{w}_{j}\right|^{2},\left|\mathbf{e}_{j} \mathbf{w}_{i}\right|^{2},\left|\mathbf{z}_{j} \mathbf{w}_{j}\right|^{2}$ and $\left|\mathbf{z}_{j} \mathbf{w}_{i}\right|^{2}$ are exponential random variables having a unitmean, which are independent of $\left|\mathbf{c}_{\kappa_{j}} \mathbf{w}_{j}\right|^{2}$, where $\chi_{v}^{2}$ is the chisquare distribution with $v$ degrees of freedom. Furthermore, $\mathbf{g}_{\kappa_{j}}$ and $\mathbf{w}_{j}$ are independent and isotropically distributed on the $N_{T}$ dimensional hyperplane, obeying the distributions $\left|\mathbf{g}_{\kappa_{j}} \mathbf{w}_{j}\right|^{2} \sim \beta\left(1, N_{T}-1\right)$ and $\left|\mathbf{g}_{\kappa_{j}} \mathbf{w}_{i}\right|^{2} \sim \beta\left(1, N_{T}-2\right)$.

$$
\begin{aligned}
\mathbf{E}\left(\left|\mathbf{h}_{j} \mathbf{w}_{j}\right|^{2}\right)= & \rho^{2} \delta\left[(1-\varphi)\left(N_{T}-N_{u}+1\right) / N_{T}+\varphi / N_{T}\right] \\
& +\rho^{2} \sigma_{e}^{2}+1-\rho^{2}
\end{aligned}
$$

with $\delta=\mathbf{E}\left(\left|\hat{\mathbf{h}}_{j}\right|^{2}\right)$ denoting the expectation of the square norm of the estimated channel vector $\hat{\mathbf{h}}_{j}$. Similarly, the expectation $\mathbf{E}\left(\left|\mathbf{h}_{j} \mathbf{w}_{i}\right|^{2}\right)$ may be calculated as:

$$
\mathbf{E}\left(\left|\mathbf{h}_{j} \mathbf{w}_{i}\right|^{2}\right)=\rho^{2} \delta \varphi /\left(N_{T}-1\right)+\rho^{2} \sigma_{e}^{2}+1-\rho^{2},
$$

where the above equation is derived by exploiting the property of $\mathbf{h}_{j} \mathbf{c}_{i}=0$, according to the ZF precoding criterion.

\section{REFERENCES}

[1] R. Zhang and L. Hanzo, "Wireless cellular networks," IEEE Vehicular Technology Magazine, vol. 5, no. 4, pp. 31-39, Dec. 2010.

[2] S. Shamai and B. M. Zaidel, "Enhancing the cellular downlink capacity via co-processing at the transmitting end," in Proc. of 53rd IEEE Vehicular Technology Conference, (VTC 2001-Spring), vol. 3, Rhodes, Greece, May 2001, pp. 1745-1749.

[3] H. Zhang and H. Dai, "Cochannel interference mitigation and cooperative processing in downlink multicell multiuser MIMO networks," EURASIP Journal on Wireless Communications and Networking, vol. 2004, no. 2, pp. 222-235, Dec. 2004.

[4] R. Zhang and L. Hanzo, "Cooperative downlink multicell preprocessing relying on reduced-rate back-haul data exchange," IEEE Trans. Veh. Technol., vol. 60, no. 2, pp. 539-545, Feb. 2011.

[5] A. A. Daoud, M. Alanyali, and D. Starobinski, "Pricing strategies for spectrum lease in secondary markets," IEEE/ACM Transactions on Networking, vol. 18, no. 2, pp. 462-475, Apr. 2010.

[6] R. Steele and L. Hanzo, Mobile Radio Communications. New York, USA: IEEE Press-John Wiley, 1999.

[7] T. S. Rappaport, Wireless Communications: Principles and Practice. Englewood Cliffs, NJ, USA: Prentice-Hall, 1996.

[8] C. K. Au-Yeung and D. J. Love, "On the performance of random vector quantization limited feedback beamforming in a MISO system," IEEE Trans. Wireless Comm., vol. 6, no. 2, pp. 458-462, Feb. 2007.

[9] N. Jindal, J. G. Andrews, and S. Weber, "Multi-antenna communication in ad hoc networks: Achieving MIMO gains with SIMO transmission," IEEE Trans. Comm., vol. 59, no. 2, pp. 529-540, Feb. 2011. 